\title{
On controlling interfacial heterogeneity to trigger bridging in secondary bonded composite joints: An efficient strategy to introduce crack-arrest features
}

\author{
Ran Tao $^{\mathrm{a}}$, Xiaole Li ${ }^{\mathrm{a}}$, AriefYudhanto ${ }^{\mathrm{a}}$, Marco Alfano $^{\mathrm{b}}$, Gilles Lubineau ${ }^{\mathrm{a}, *}$ \\ a King Abdullah University of Science and Technology (KAUST), Physical Sciences and Engineering Division (PSE), COHMAS Laboratory. Thuwal, 23955-6900, Saudi Arabia \\ ${ }^{\mathrm{b}}$ Department of Mechanical, Energy and Management Engineering, University of Calabria, 87036, Rende (CS), Italy
}

\section{A R T I C L E I N F O}

\section{Keywords}

Adhesive joints

CFRP

Interface

Crack-arrest feature

Cohesive zone model

\begin{abstract}
A B S T R A C T
Secondary adhesive bonding of carbon fiber reinforced polymer laminates is of great interest for the aerospace and automotive industries. However, joint reliability is still a major concern because of fabrication-induced or service-related cracks that are difficult to monitor and can lead to catastrophic failure. In this work, we propose a patterning strategy where the careful design of spatially-varying interface properties enables the formation of an adhesive ligament that acts as a crack-arrest feature. Bi-dimensional finite element models of adhesively bonded double cantilever beams were employed to investigate the role of the main parameters of the pattern ( i.e., geometrical parameters and interfacial properties) under mode I loading. The results show that an adhesive ligament can either bridge the separating arms, largely enhancing the dissipated energy, or fail, thereby limiting the attainment of a R-curve-like response. The precise scenario is heavily dependent on the contrast in interfacial properties rather than the geometrical details of the pattern.
\end{abstract}

\section{Introduction}

Carbon fiber-reinforced polymers (CFRPs) have been widely adopted in aerospace structures and have recently received increased attention in the automotive industry $[1,2]$. However, joining cured composite materials with structural adhesives (i.e., secondary bonding) still remains a major challenge. Conventional mechanical fastening techniques, such as riveting or bolting, are still widely used but result in a large increase in weight and cost. Rivets/bolt-free secondary adhesive bonding present an ideal solution for joining CFRPs, enabling massive weight reduction, more integrated structures, and uniform stress distribution over the joint region [3-8]. Yet, we are still far from such widespread industrial application of adhesive joints. The most important concern preventing the practical application of adhesively-bonded composite joints for primary load bearing structures arises from the fact that any premature delamination is difficult to detect, and once the crack is initiated, brittle catastrophic failure occurs. Thus, it is crucial to introduce crack arresting features that can slow down (or even stop) crack growth and improve damage tolerance of composite joints. While the absolute intrinsic performance of the joint is important, limiting the progression of any damage is more crucial, to ensure confidence in the design and allow enough time for maintenance and repair.
Several methods have been reported to delay or arrest crack propagation. Tserpes et al. manufactured CFRP adherents with surface corrugation and observed a crack-arrest effect in double cantilever beam (DCB) tests [9]. A higher fracture load was required because of mode transition from opening to shear stress. Despite the significant enhancement, the use of corrugations is still limited, because the shape and the geometry of the structures are usually constrained based on the intended application. The systematic corrugation of the substrate also might not be feasible, especially in CFRP where carbon fibers restrict the geometrical perturbations.

Z-pinning and stitching is a more popular crack-arrest feature implemented for impeding the growth of delamination in composites [10-12]. Carefully designed through-thickness reinforced fibrous pins could largely improve the ultimate shear strength of composite single lap joints by enabling extrinsic energy dissipation [10]. However, through-thickness reinforcements can only be applied in co-cured joints, and even in such cases, they can be detrimental to the substrate because of the wavy fibers, which increases fiber breakage events [12]. In any case, z-pinning or stitching cannot be applied in the context of secondary bonding where the parts to be joined together are already cured.

Modifying or structuring the adhesive layer or its interface with the substrate is an alternative way to introduce crack-arrest features, as opposed to the strategies described above which target the bulk of

\footnotetext{
${ }^{*}$ Corresponding author.

E-mail address: gilles.lubineau@kaust.edu.sa (G. Lubineau)
} 
the substrates. One example suggested co-bonding an extra continuous fiber layup inside the adhesive layer, which was manufactured by interlocked $0^{\circ}$ layers [13]. When the crack passed through the crossing part of the above arrest feature in DCB tests, a higher applied load was required for further growth [13]. However, delamination was found to possibly occur at the interfaces between the interlocked layer and composite substrates without triggering the embedded crack-arrest feature. Another way to structure the adhesive layer has been suggested by co-curing thermoplastic patches with CFRP substrates during the fabrication process. These thermoplastic films were aligned and welded when applying epoxy adhesive to bond the joint [14]. This integration of the thermoplastic inclusions successfully trapped the crack growth and largely enhanced the mechanical strength [14]. However, co-curing and welding of thermoplastic patches require extra manufacturing steps; moreover, the adhesion between thermoplastic material and epoxy needs to be carefully monitored.

New concepts for enabling crack arrest by triggering adhesive ligaments have been discussed in the authors' previous research $[8,15]$. A laser-based surface patterning strategy for CFRP substrates was proposed to generate adhesive ligaments and promote extrinsic energy dissipation during the fracture process [8]. Further work by the authors, enabled by insitu analysis of the damage, illustrated that the adhesive ligaments were randomly triggered because of the highly heterogeneous surface of the laser-treated CFRPs [15]. However, a detailed investigation is needed on the mechanism controlling the formation of adhesive ligaments to efficiently design interface patterns and tailor energy dissipation and fracture toughness. The R-curve response associated with the extrinsic bridging phenomena was also reported in Refs. [16,17], where the adhesive layer was reinforced by either a plastic or metallic carrier. Heide-Jørgensen et al. analyzed the R-curve due to bridging of a nylon carrier embedded in a commercial epoxy adhesive film to set the layer thickness, and found a significant toughness enhancement [16]. In Maloney and Fleck's work, non-sticky PTFE films were alternatively placed at the top and bottom of $\mathrm{Al} /$ adhesive interfaces to trigger bridging of the adhesive layer reinforced with a copper mesh, which significantly enhanced the joint fracture toughness [17]. In addition, previous works have shown that bio-inspired design of T-joints with composite adherents exhibit an improved toughness thanks to the extra energy dissipation associated to crack branching and crack bridging $[[18,19]$. Thus, triggering adhesive ligaments (with or without reinforcement within the adhesive layer) can be an effective alternative crack-trapping strategy relative to other techniques, e.g., corrugation [9], z-pinning [10], stitching [11], and thermoplastic inclusions [14]. Indeed, triggering adhesive ligaments only relies on modification of the mating surfaces or the property of the adhesive, without additional modifications within the substrates.

In this work, a patterning strategy was proposed to introduce a crack-arrest feature into an adhesively bonded composite joint, in the form of adhesive ligaments. Recent works have shown that patterned interfaces may have a profound effect on the mechanical behavior of bonded joints [20-26]. Because of spatially varying adhesion properties, important variables such as fracture toughness [20,22,23,26], stability and kinetics of crack growth [24,25], as well as the crack path within the joint $[21,26]$, can be largely affected. In this work we use a patterning strategy as a mean to control the mechanism of crack propagation with an adhesive joint comprising composite adherents. By tailoring adhesion properties of the mating surfaces, we aim to deflect an incoming interfacial crack by promoting a jump from the lower to the upper interface (or vice versa). In the process, an adhesive ligament can be generated that bridges the crack faces, hinders crack propagation, and leads to an increase in the overall energy dissipation.
In this work, two regions with strong adhesion, i.e., adhesion strength and fracture toughness, were alternatively patterned on the top and bottom CFRP/adhesive interfaces to facilitate the formation of an adhesive ligament. The proposed patterning strategy and the toughening effect were assessed through computational simulations of crack growth performed in the FE framework using cohesive zone models and considering the double cantilever beam (DCB) configuration. The objective of this work is to understand the formation of the adhesive ligament and to investigate how the associated dissipation is affected by the geometry and surface properties of the interfacial pattern. Since hindering the propagation of incoming cracks is crucial for the reliability of layered structures, our aim is to engineer the response of the adhesive layer and promote a R-Curve response, where the fracture resistance increases as the crack propagates.

The manuscript is organized as follows. First, a 2D finite element model of DCB tests is introduced and validated by comparing with the conventional single cohesive layer model and the well-known related analytical solution. Then, the choices of cohesive strength and cohesive fracture energy values are determined based on the authors' previous experimental investigations. Finally, the formation of the adhesive ligament and the enhanced energy dissipation are analyzed by varying the anchor size, gap distance, cohesive strength, and cohesive fracture energy.

\section{Methods \\ 2.1. Modeling approach}

A 2D finite element model of a DCB specimen was adopted in this study to assess the R-curve response associated with the formation of an adhesive ligament. In conventional simulation models, the adhesive layer and interfaces are usually replaced by a single layer of cohesive elements, whose parameters are determined using experimental calibration $[27,28]$. This concept of simulation model is rooted in the finite-element study of the delamination of CFRP laminates [29-32], where the fractured surface can be viewed as zero-thickness mid-plane. However, it does not allow to account for the complex synergy of local and non-local energy dissipation within the adhesive layer during fracture, which may be significant when using ductile adhesives or a reinforcing third phase. Instead of employing the cohesive zone model, Wei et al. [33] proposed a numerical model using elastic interface properties to capture the stress distribution along the adhesive thickness. In order to include the non-local contributions, researchers have developed modeling approaches that explicitly account for the bridging fibers [34] or the adhesive layer [35-37]. Thus, to investigate the mechanisms controlling the formation of an adhesive ligament (which, in essence, corresponds to a non-local mechanism of dissipation [30]), the adhesive layer was fully modeled as a volume entity and sandwiched between cohesive elements, which mimicked a debonding at the top and bottom interfaces. In addition, bulk plasticity within the adhesive layer was also accounted for by introducing isotropic hardening prior to final failure at a critical strain level. Two arrest regions, i.e., anchors, with given length $b$ (i.e., anchor size) and strong adhesion, were alternatively patterned on the top and bottom baseline CFRP/adhesive interfaces. The anchors were separated by a gap distance $g$. Here, the crack was always initiated on the bottom interface and the arrest features were located far enough from the crack initiation to ensure that the initial response was not perturbed (see Fig. 1). This corresponded to what was expected from a classical DCB test in which the interface had the baseline properties. Both baseline and arrest interfaces are represented by a bilinear cohesive zone model. 


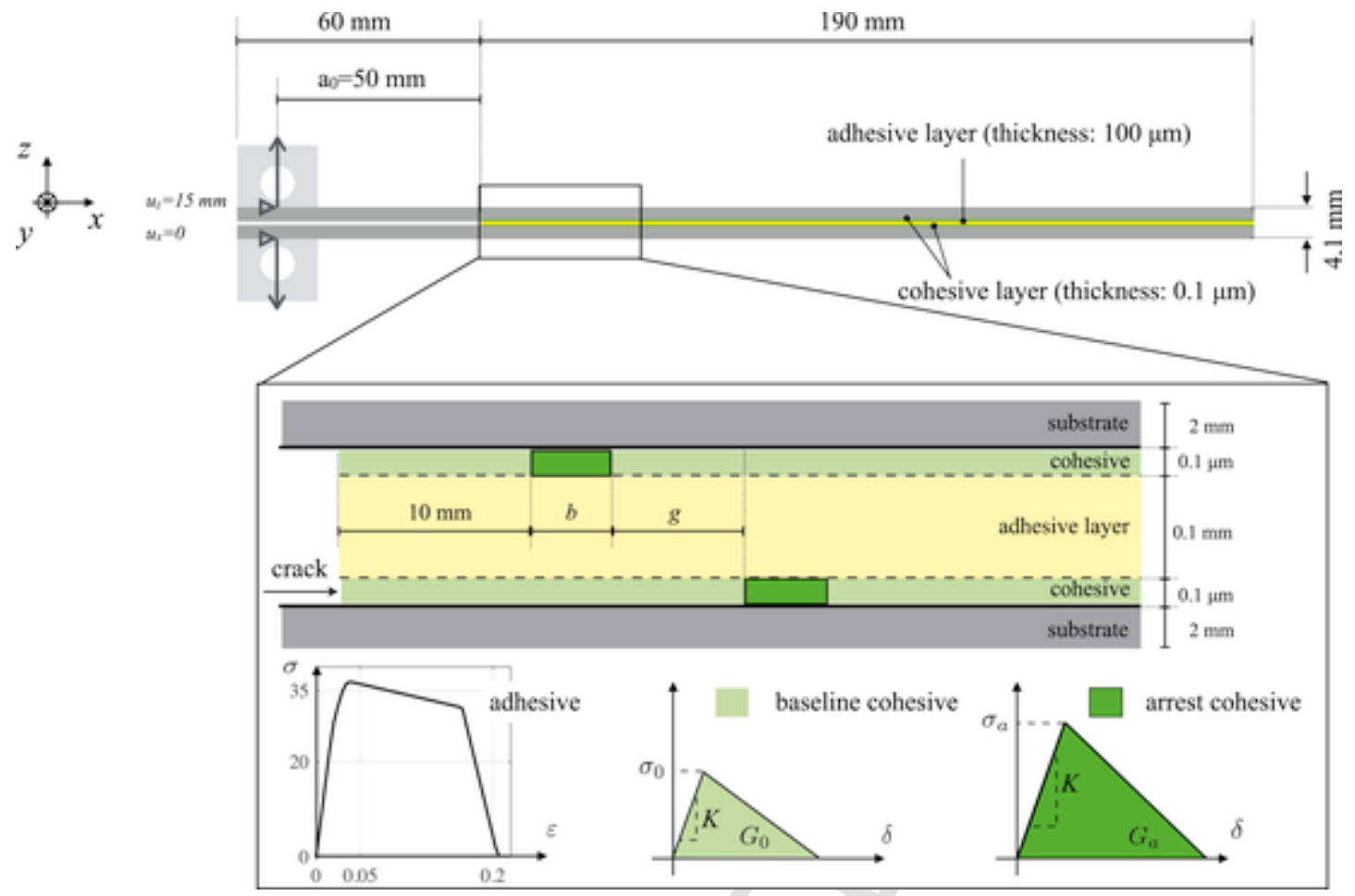

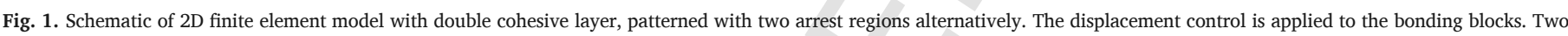

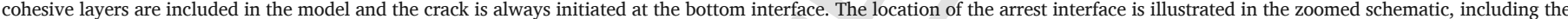
stress-strain curve of the adhesive layer and the traction-separation relations of both the baseline and arrest interfaces.

\subsection{Model dimensions and input properties}

The total dimensions (length by width by thickness) of the DCB specimen were $250 \times 20 \times 4.1 \mathrm{~mm}^{3}$, and the pre-crack distance $a_{0}=50 \mathrm{~mm}$ , as shown in Fig. 1. The substrate and adhesive materials were extracted from the authors' previous experimental work. The substrate was a 2-mm thick unidirectional T700/M21 CFRP laminate, which was assumed orthotropic and linear elastic, with $E_{x x}=125000 \mathrm{MPa}$, $E_{y y}=E_{z z}=7800 \mathrm{MPa}, v_{x y}=v_{x z}=0.33, v_{y z}=0.4, G_{x y}=G_{x z}=5100 \mathrm{MPa}$, and $G_{y z}=2786 \mathrm{MPa}$. The adhesive layer was made of a two-component epoxy adhesive paste (Araldite $420 \mathrm{~A} / \mathrm{B}$, Huntsman, Salt Lake City, UT, USA) with a thickness of $t_{a}=100 \mu \mathrm{m}$. The initial behavior was isotropic and linear elastic ( $E=1500 \mathrm{MPa}$ ) followed by bulk plasticity up to failure at a critical strain. In particular, after reaching the yield strength of $\sigma_{y}=27 \mathrm{MPa}$, the adhesive layer experienced isotropic hardening until it achieved the ultimate strength $\sigma_{\max }=37 \mathrm{MPa}$ at plastic strain $\varepsilon_{p}=1.2 \%$ . Linear softening was then prescribed during the post-peak regime until final failure at $\varepsilon_{\text {fail }}=20 \%$, as shown in Fig. 1. This specific adhesive property mimicked that reported in the product data-sheet and related literature [38]. Both substrates and the adhesive layer were discretized using plane strain Q4 elements with reduced integration and enhanced hourglass control. Rigid discrete element was used for the bonding block, where the displacement control was prescribed $\left(u_{x}=0\right.$, $u_{z}=15 \mathrm{~mm}$ ), as shown in Fig. 1. This DCB specimen was solved using the standard/static solver while accounting for the geometrical non-linearity (NLGEOM) within ABAQUS/CAE 2017 (ABAQUS, Simulia, Johnson, RI, USA).

Two layers of bi-linear cohesive elements were used at the top and bottom CFRP/adhesive interfaces, with a thickness of $0.1 \mu \mathrm{m}$. The baseline cohesive strength $\left(\sigma_{0}\right)$ and cohesive fracture energy $\left(G_{0}\right)$ characterized the behavior of the baseline interface, while higher cohesive strength and cohesive fracture energy $\left(\sigma_{a}\right.$ and $G_{a}$ ) were given to the arrest interfaces, (Fig. 1). Weak interfacial properties ( $\sigma_{i}=1 \mathrm{MPa}, G_{i}=0.01 \mathrm{~kJ} / \mathrm{m}^{2}$ ) were assigned to the first cohesive element on the bottom interface to ensure that the initiation always started from the bottom. The crack path was expected to be diverted by this local heterogeneity from the initial bottom interface to the top interface and thus trigger the formation of a single adhesive ligament. Since the adhesive ligament could locally lead to mode mixity, a built-in maximum stress initiation criterion is used, assuming the same critical stress for each mode. When one of the normal stress or shear stress reaches the critical value, damage initiates within the cohesive element. A built-in mixed-mode law, with one critical value of the total fracture energy governing the sum of the mixed-mode energy, is employed to characterized the evolution of cohesive failure. As for the interfacial properties, cohesive strength and cohesive fracture energy of the baseline interface $\left(\sigma_{0}\right.$, $G_{0}$ ) and the arrest interface ( $\sigma_{a}, G_{a}$ ) were determined based on the authors' previous experimental investigations $[8,15]$, in which the same CFRP laminate and epoxy adhesive were employed.

Fracture toughness values under DCB tests of different uniform surface pretreatments are listed in Table 1 [8]. A toughness range between $0.15 \mathrm{~kJ} / \mathrm{m}^{2}$ and $1.50 \mathrm{~kJ} / \mathrm{m}^{2}$ was considered in this work, which corresponded to values that can be achieved experimentally with various pretreatments. The baseline fracture toughness was set to be

Table 1

Interfacial toughness values of different surface pretreatments based on DCB tests [8]. PP : peel-ply; $L A$ : laser ablation; $L C$ : laser cleaning; $S B$ : sandblasting; $S$ : sanding.

\begin{tabular}{ll}
\hline Surface & $G\left(\mathrm{~kJ} / \mathrm{m}^{2}\right)$ \\
\hline$P P$ & 0.17 \\
$L A$ & 0.40 \\
$L C$ & 0.75 \\
$S B$ & 1.13 \\
$S$ & 1.60 \\
\hline
\end{tabular}


$G_{0}=0.15 \mathrm{~kJ} / \mathrm{m}^{2}$, and the toughness of the arrest interface $G_{a}$ varied between $0.15,0.18,0.30,0.45,0.60,0.75$, and $0.90 \mathrm{~kJ} / \mathrm{m}^{2}$. These choices led to a toughness ratio $G_{a} / G_{0}$ within the range $1 \sim 6$. As stated earlier, the core purpose of the proposed strategy is to hinder and, possibly, arrest crack propagation. The chosen set of cohesive properties for the baseline interface provides more flexibility in exploring the potential crack arrest mechanisms. Note that, the fracture toughness of the Araldite 420 epoxy adhesive is around $3 \mathrm{~kJ} / \mathrm{m}^{2}$ [38], which is larger than the values at the CFRP/adhesive interfaces. Since the adhesive layer is tougher than the CFRP/adhesive interfaces, the classic scenario of cohesive failure within the adhesive layer could not be reached in this configuration. The proposed approach provided solutions for improving the performance of joints even when uniform surface pretreatments were not sufficiently efficient to achieve the best of the used adhesive. Finally, adhesion strength obtained from three-point bending of miniature single-lap joints are listed in Table 2 [15], and thus the baseline value was fixed as $\sigma_{0}=15 \mathrm{MPa}$ and that of the arrest interface was 15, 15.1, 15.2, $15.3,15.4,15.5,16,16.5,18,21$, and $24 \mathrm{MPa}$. These choices led to a strength ratio $\sigma_{a} / \sigma_{0}$ within the range $1 \sim 1.6$.

Based on previous experimental investigations, these combinations could be realized experimentally and could make a viable design space for the baseline and arrest properties. Regarding the geometry of the pattern, the anchor size $(b)$ was set to vary between 1,5 , and $10 \mathrm{~mm}$, while the gap distance $g$ was changed from $0,1,5,10$, and $20 \mathrm{~mm}$, inspired from previous experimental investigations.

\subsection{Model validation}

The simulation model was firstly validated by conducting a sensitivity study of the mesh and viscous regularization values. The viscous regularization value was employed to improve the rate of convergence without compromising the results. The corresponding load-displacement curves are shown in Fig. 2. The responses were unreli-

Table 2

Adhesion strength values of different surface pretreatments based on three-point bending tests [15]. $P P$ : peel-ply; $L A$ : laser ablation; $T$ : Teflon; $L C$ : laser cleaning; $S$ : sanding.

\begin{tabular}{ll}
\hline Surface & $\sigma(M P a)$ \\
\hline$P P$ & 15.0 \\
$L A$ & 19 \\
$T$ & 21.5 \\
$L C$ & 24.5 \\
$S$ & 29.0 \\
\hline
\end{tabular}

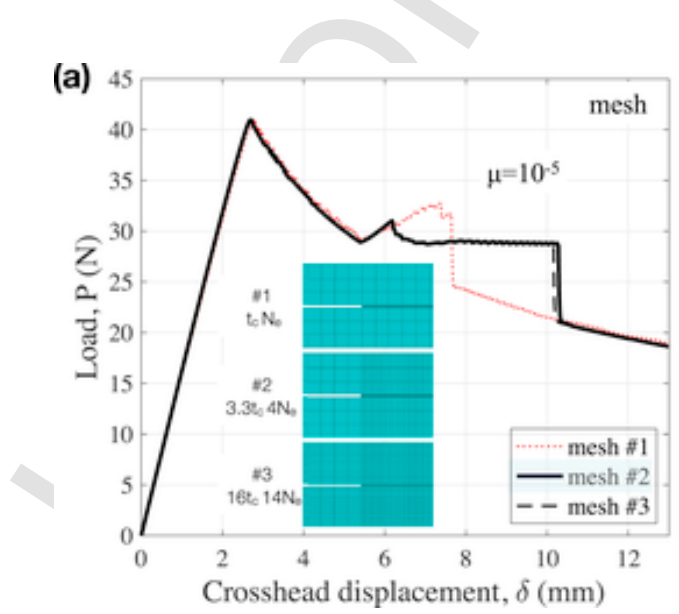

able when using coarse mesh and large viscosity, such as mesh \#1, $\mu=10^{-3}$, and $\mu=10^{-4}$. On the other hand, using a fine mesh (mesh \#3) or small viscosity value $\left(\mu=10^{-6}\right)$ incurred large computational costs but led to results with similar accuracy. Thus, an optimized mesh density and viscous regularization value, mesh $\# 2$ and $\mu=10^{-5}$, were deployed in this simulation study.

Then, comparisons among the proposed model, a conventional numerical DCB model with a single cohesive layer, and the analytical solution were performed to further validate the proposed approach. Two simulation cases were considered, i.e., uniform interfaces and patterned interfaces with aligned arrest regions, as shown in the schematic in Fig. 3. The proposed double cohesive model and the conventional single cohesive model demonstrated identical load-displacement curves, which were the same as the analytical solution, although the analytical model deviated slightly at the transition point due to its assumption of infinite adhesion strength. Thus, the proposed double cohesive model could replace the conventional DCB model in these simulation cases. In addition, the proposed simulation approach gives more flexibility and it is promising to investigate complex patterning strategies that enable the formation of adhesive ligaments such as that proposed in this work.

In order to obtain the R-curve of simulated DCB tests, a homemade python script was employed to capture the crack length $a$. The crack length is defined as the distance between the loading points shown in Fig. 1 and the current crack front (where the damage variable $d$ starts to be lower than 1) at corresponding opening displacement $\delta$. The compliance calibration (CC) method, suggested by the standard ASTM D552813 [39], was chosen for the calculation of the energy release rate (ERR) $G_{I}$ :

$G_{I}=\frac{n P \delta}{2 b a}$,

where $P$ is the applied load, $b=20$ is the specimen width, and $n$ is the CC term, which was extracted from a least-square fitting of the logarithmic compliance $(C=\delta / P)$ against the logarithmic crack length, i.e., $\log (C)$ $\log (\mathrm{a})$.

\section{Results}

\subsection{Typical crack-arrest mechanisms}

Three typical crack-arrest mechanisms were observed through the simulations, which were grouped as $N L$ (no ligament), $U L$ (unbroken ligament), and $B L$ (broken ligament), as illustrated in Fig. 4. Each scenario corresponds to a drastically different macroscopic load-displacement curve. The differences among all cases are detailed below,

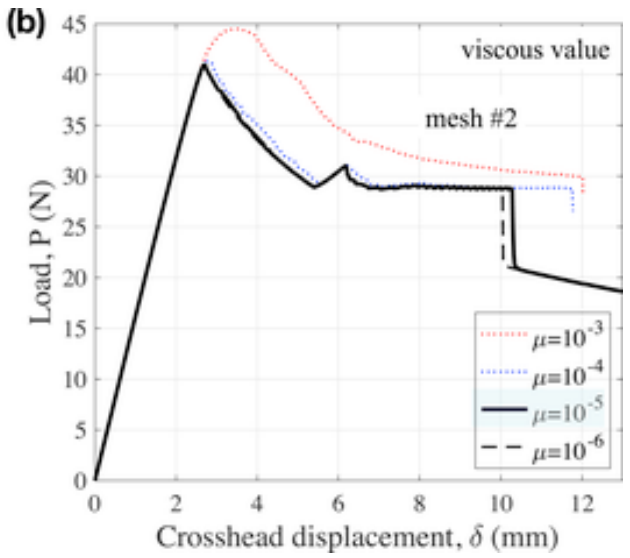

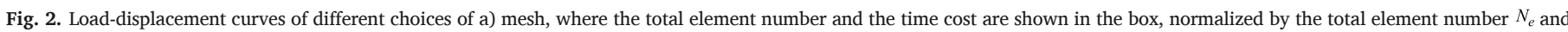
the time cost $t_{c}$ of the mesh\#1, and $\mathrm{b}$ ) viscous regularization values. 
(a)
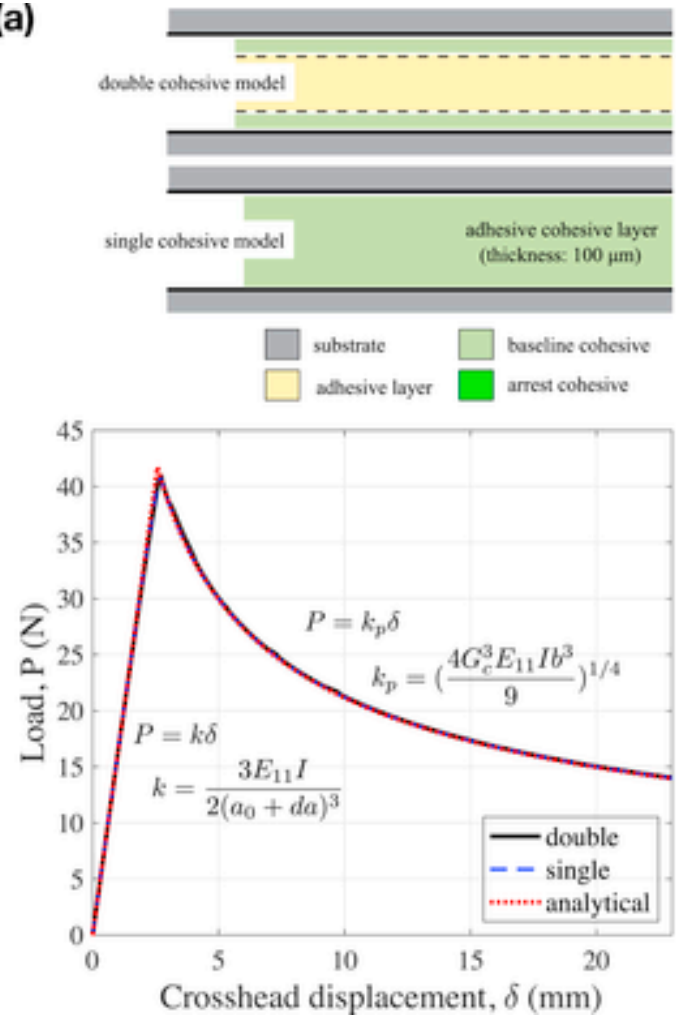

(b)
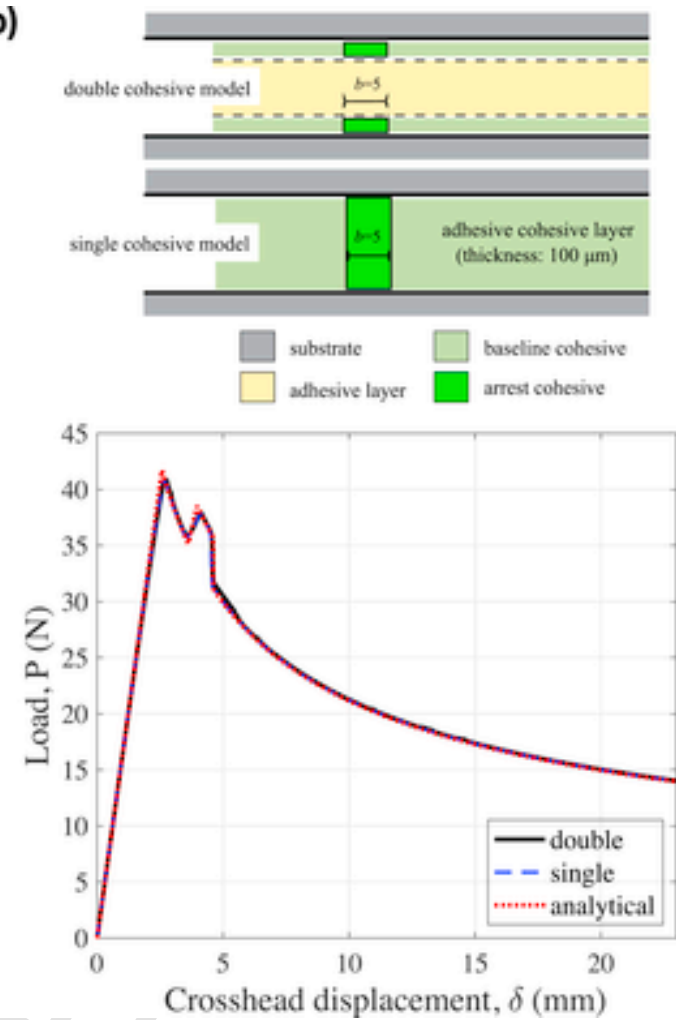

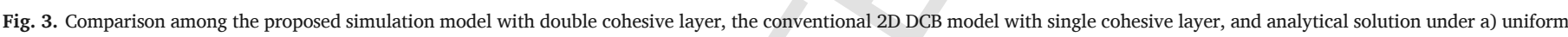

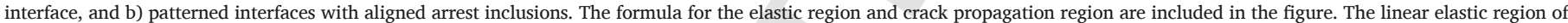
the analytical solution is corrected by a virtual crack advance $d a$ due to the end edge rotation [41], where $d a=h \sqrt{\frac{E_{11}\left(3-2(\gamma /(1+\gamma))^{2}\right.}{11 G_{12}}}$ and $\gamma=1.18 \sqrt{\frac{E_{11} E_{22}}{G_{12}}}$.

$N L$, no ligament

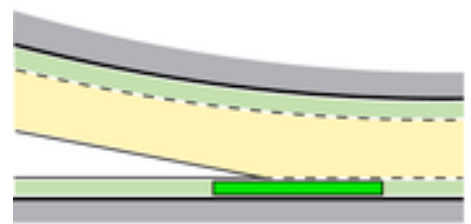

$U L$, unbroken ligament

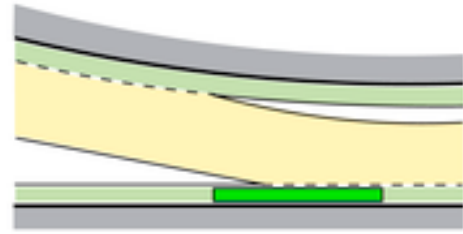

substrate adhesive layer
$B L$, broken ligament

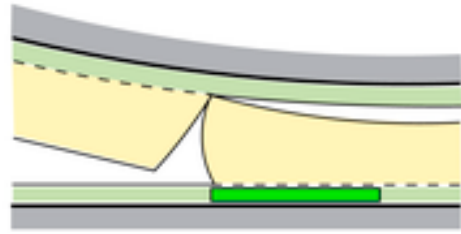

arrest cohesive

Fig. 4. Schematics of three typical crack-arrest mechanisms: $N L, U L$, and $B L$.

and the controlling parameters (mainly cohesive strength and cohesive fracture toughness) will be discussed in the following section.

The typical responses for the cases discussed above, i.e., $N L, U L$, and $B L$, are illustrated in Fig. 5, Fig. 6, and Fig. 7, respectively. All three figures include the R-curve, energy decomposition obtained from ABAQUS historical output, snapshots of the crack propagation, load-displacement curve, and the schematic of the crack front. $G_{0}$ and $G_{a}$ represent the R-curve and load-displacement response of the uniform baseline interface and uniform arrest interface, respectively. $E_{c}$ is the fracture energy dissipated from the cohesive elements and $E_{a}$ is the sum of the plastic and fracture energy of adhesive elements, which both vary versus the crack length $a$. The initial crack length, $a_{0}=50 \mathrm{~mm}$, is the start point of $x$-axis in the figures. For all three plots, $b=5 \mathrm{~mm}, g=10 \mathrm{~mm}$, and $G_{a}=0.18 \mathrm{~kJ} / \mathrm{m}^{2}$ are fixed, while the cohesive strengths are $\sigma_{a}=15$, $\sigma_{a}=16$, and $\sigma_{a}=24 \mathrm{MPa}$, respectively. The regions highlighted in blue, located at $60<a<65$ on the top interface and $75<a<80$ on the bottom in the figures, illustrate the location of the arrest region.
In $N L$, when the process zone front ( $d=0$, blue triangle in Fig. 5 (a)) reached the arrest region (point 1), the ERR $G_{I}$ increased to the value of the arrest region. The applied load also increased accordingly, as shown in Fig. 5 (b). When the crack tip ( $d=1$, blue circle in Fig. 5 (a)) reached the arrest region (point 2), the crack propagated again along the arrest region, as illustrated by the schematic in Fig. 5 (c). Between point 2 and 3 , the ERR value reached that of arrest region $G_{a}$. Then the crack passed the arrest region and the ERR dropped back to the baseline value after point 3, which can be also observed from the load-displacement curve in Fig. 5 (b). Thus, since no ligament was triggered, the delamination occurred at the bottom interface and the R-curve response was limited and the energy dissipated from the adhesive layer $E_{a}$ was zero. The mechanical responses of $N L$ were similar to the conventional simulation model using a single cohesive layer.

In the cases $U L$ and $B L$, the adhesive ligament was triggered after the crack tip reached the arrest region and tried to detach from the bottom arrest interface (point 2 in Figs. 6 (a) and Fig. 7 (a)). Since 

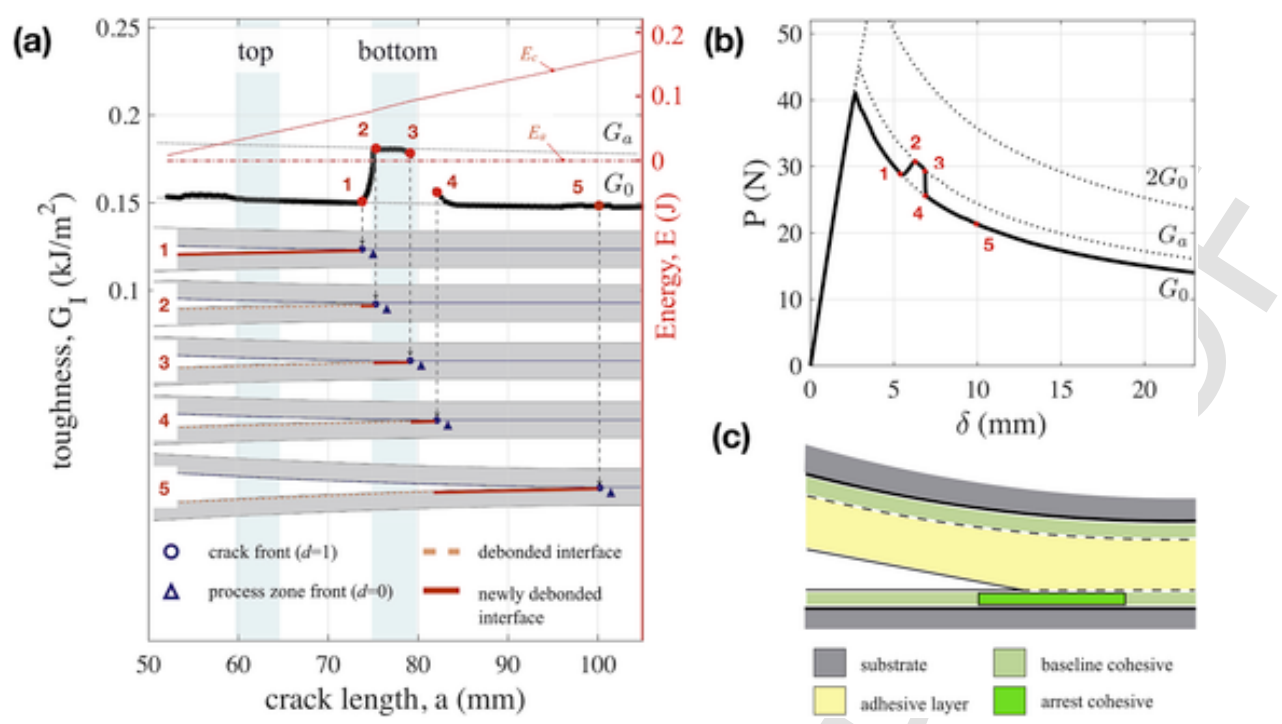

(c)

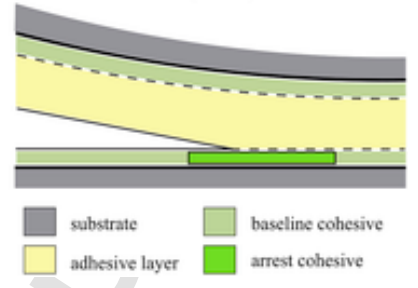

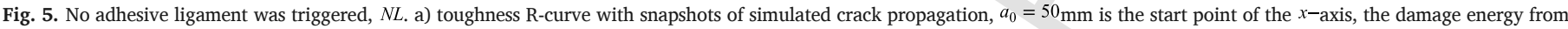

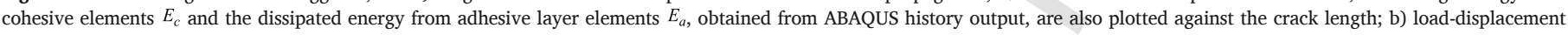
curve; c) schematic of the crack-arrest mechanism.
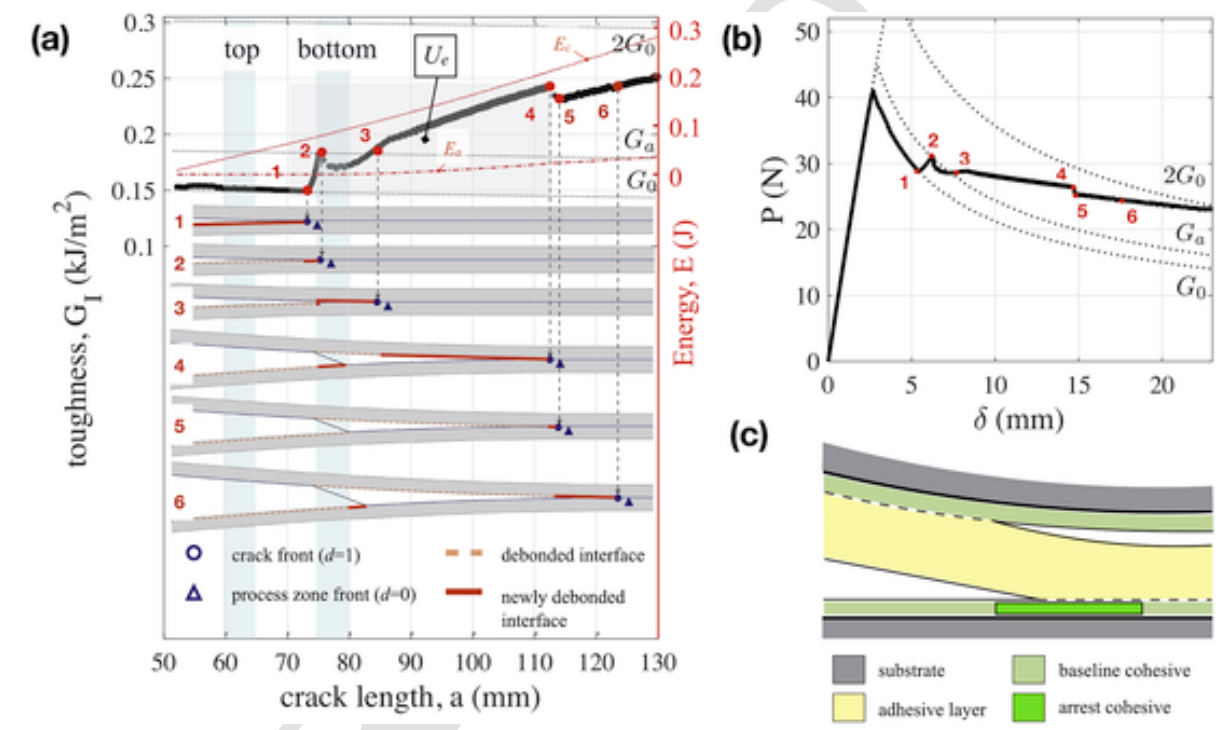

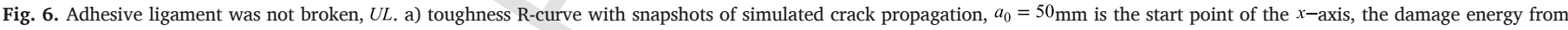

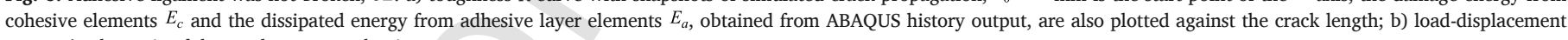
curve; c) schematic of the crack-arrest mechanism.

the delamination transferred to the top interface, which had lower strength and toughness, a short period of unstable propagation occurred (point 3). After this point, $U L$ and $B L$ displayed different behavior.

In $U L$, the triggered ligament could be peeled off from the arrest region (between point 3 and point 4) due to relatively low strength and toughness of the arrest region. Both the ERR and applied load slightly decreased at point 4 because the delamination of the ligament transited from the arrest region $G_{a}$ to the baseline interface $G_{0}$ . Then, after point 4 , as the crack propagated along the top interface, the ligament was peeled off from the bottom interface. The R-curve shows that the ERR tends to be double the baseline value, i.e., $2 G_{0}$ , and the load-displacement response approaches the response of a homogeneous interface with toughness $2 G_{0}$. Since the adhesive ligament kept stretching, the energy dissipation of the adhesive layer increased as the crack propagated. The major energy dissipation in the adhesive was the plastic energy, which was $98.7 \%$ of $E_{a}$. The ratio between $E_{a}$ and $E_{c}$ reached a maximum value of $13.1 \%$ at point 4 . The crack-arrest mechanism of $U L$ is illustrated in the schematic in Fig. 6 (c), showing that the triggered adhesive ligament detaches from the bottom arrest region and the crack propagates at both CFRP/adhesive interfaces. A similar phenomenon of the bridging adhesive layer was reported in Budzik's work in composite/aluminum joints [21].

On the other hand, in $B L$, instead of peeling off from the arrest region, the adhesive ligament detached from the baseline top interface, shown as the left part of the newly debonded interface in snapshot 4 in Fig. 7 (a). Between point 3 and 4, the ligament detached slowly from the top interface and the detached distance determined the length of the adhesive ligament. As the detachment and gradual stretching of the ligament continued, the ERR kept increasing and even exceeded the value of the arrest region $G_{a}$. Due to the large cohesive strength of the arrest region, the adhesive ligament was stretched to final failure before detaching from the bottom interface 

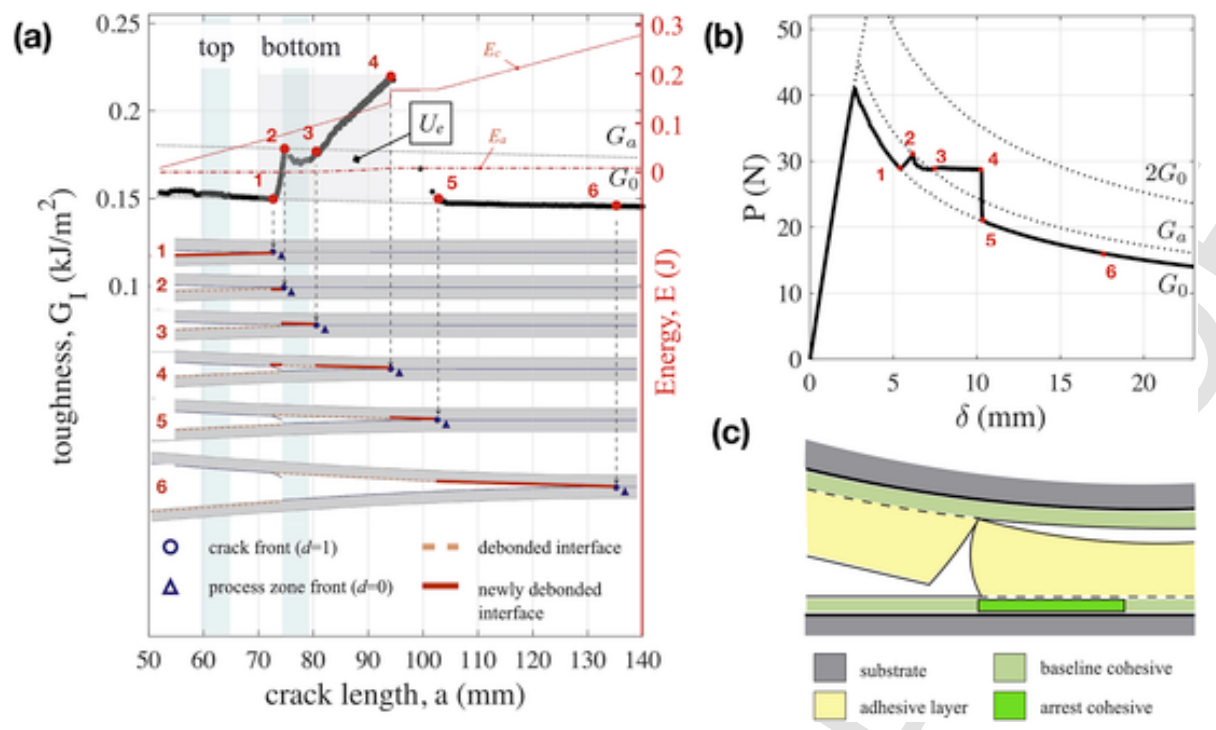

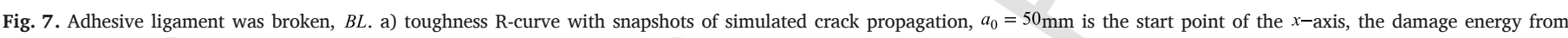

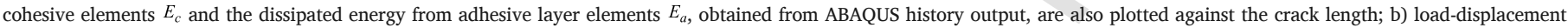
curve; c) schematic of the crack-arrest mechanism.

(point 4). The dissipated energy of the adhesive layer increased before the failure of the adhesive ligament, with a maximum ratio $E_{a}=5.6 \% E_{c}$ . The fracture energy dissipation from the adhesive layer was limited, which was only $2.1 \% E_{a}$. After the failure of the ligament, the crack propagated along the top baseline interface and both the R-curve and the load-displacement curve dropped back to the baseline values, and the plastic energy of the adhesive layer kept unchanged. Compared to $N L$, which failed to trigger the ligament, $B L$ arrested the crack advance for a larger crosshead opening $\delta$ and enhanced ERR because of the presence of the ligament. The crack-arrest mechanism of $B L$ is illustrated in the schematic in Fig. 7 (c), showing that the triggered adhesive ligament breaks before detaching from the bottom arrest region.

\subsection{Parametric study}

Parametric study of different anchor size $b$, gap distance $g$, cohesive strength $\sigma_{a}$ and cohesive fracture energy $G_{a}$ of the arrest region was performed. The main objective was to determine the effect of the geometrical parameters and the contrast in interfacial properties on the formation of ligaments, as well as on the enhancement in dissipated energy. This information is required to ensure that crack-ar- rest features created by surface patterning could become practically controllable and eventually become a viable technology. The simulation results are summarized below.

The map in Fig. 8 shows which of the three different crack-arrest mechanisms, $N L, U L$, and $B L$, is activated depending on the cohesive strength ratio $\left(\sigma_{a} / \sigma_{0}\right)$ and cohesive fracture energy ratio $\left(G_{a} / G_{0}\right)$. Note that both the strength ratio and toughness ratio were larger than 1 because the arrest region was, by convention, stronger and tougher than the baseline interface. The strength ratio is the main parameter that guides the triggering of ligaments: a small strength ratio does now allow the formation of an adhesive ligament ( $N L$ ) (Fig. 8 (a)). On the other hand, the toughness ratio plays a critical role in distinguishing $U L$ and $B L$. When $G_{a} / G_{0}>1.4$, the triggered ligament breaks before detaching from the arrest region ( $B L$ ) (Fig. 8 (a)), whereas a small strength ratio $\sigma_{a} / \sigma_{0}<1.5$ is needed when $G_{a} / G_{0}<1.4$, to maintain an unbroken adhesive ligament $(U L)$, as illustrated in Fig. 8 (b). Fig. 8 (a) shows only the magnified part, highlighted by the red box in Fig. 8 (b), illustrating the strength-toughness map for the formation of the adhesive ligament. Three typical crack-arrest mechanisms $(N L, U L$, and $B L)$ are schematically depicted in Fig. 4; note that these specific values of the strength and toughness ratio can be varied based on the geometrical dimensions
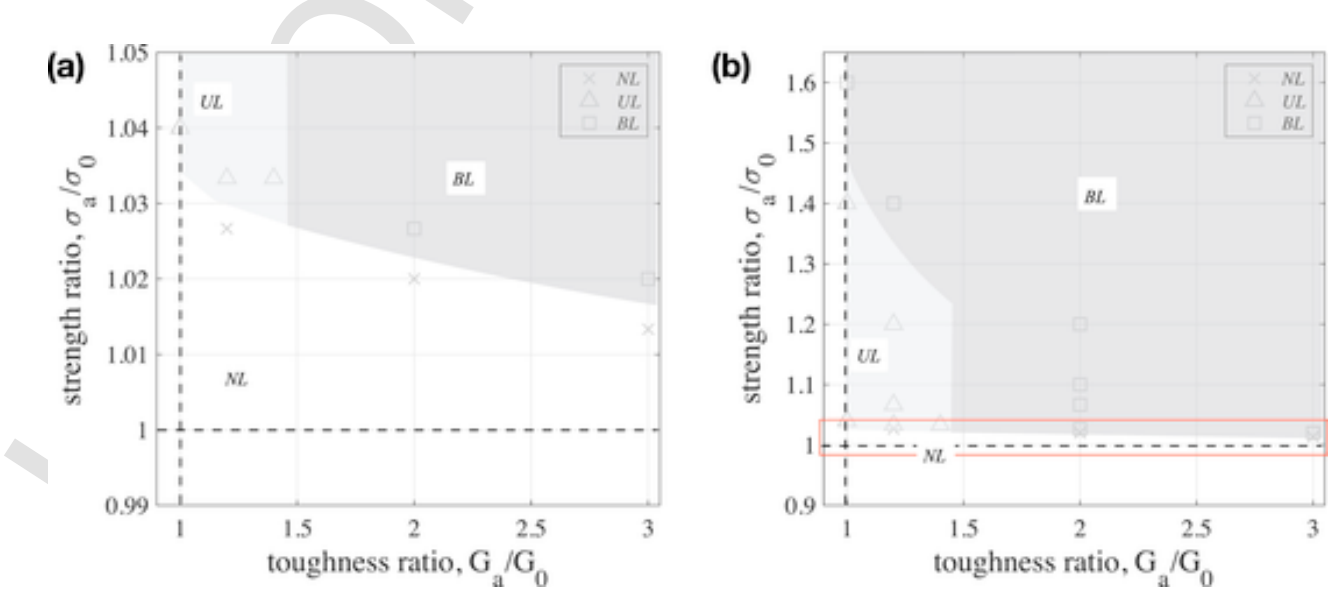

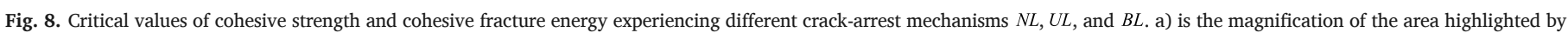
the red box in b). (For interpretation of the references to colour in this figure legend, the reader is referred to the Web version of this article.) 
and material properties of the adhesive layer. A thick adhesive layer needs a larger strength and toughness ratio to trigger an adhesive ligament, which agrees with the previous discussion by Sills that the cohesive strength ratio increases with increasing crack-jump distance [32]. Extra simulation work was carried out, where lower ultimate maximum stress was applied to the adhesive layer, which narrowed the range of choice of the strength and toughness ratios in the case of unbroken ligament $U L$ in Fig. 8. Increasing the failure stress of the adhesive material, in order to push the boundary of the $U L$ domain further to the right, thus allowing a broader design space for practical applications, presents an interesting possibility for future study.

Further simulation results concerning the effect of the geometrical parameters of the pattern and strength-toughness ratios on the saftety of adhesive joints, which includes the extra dissipated energy $\left(U_{e} / U_{0}\right.$ ) and the peak energy release rate $\left(G_{\max } / G_{0}\right)$, are shown in (Fig. 9) and (Fig. 10). The extra dissipated energy $U_{e} / U_{0}$ corresponds to the required energy during the whole crack arresting process, while the peak ERR $G_{\max } / G_{0}$ represents the maximum resistance to the fracture of the joint in service. To obtain the extra dissipated energy, the total dissipated energy $U$ was calculated by integrating the R-curve from $a=70$ $\mathrm{mm}$ until total detachment from the arrest region in $U L$ (point 4, highlighted in Fig. 6 (a)) or from $a=70 \mathrm{~mm}$ until failure of the adhesive layer in $B L$ (point 4, highlighted in Fig. 7 (a)). The baseline dissipated energy $U_{0}$ was calculated along the same crack length with baseline toughness $G_{0}$. Thus, the extra dissipated energies were obtained by subtracting the baseline values
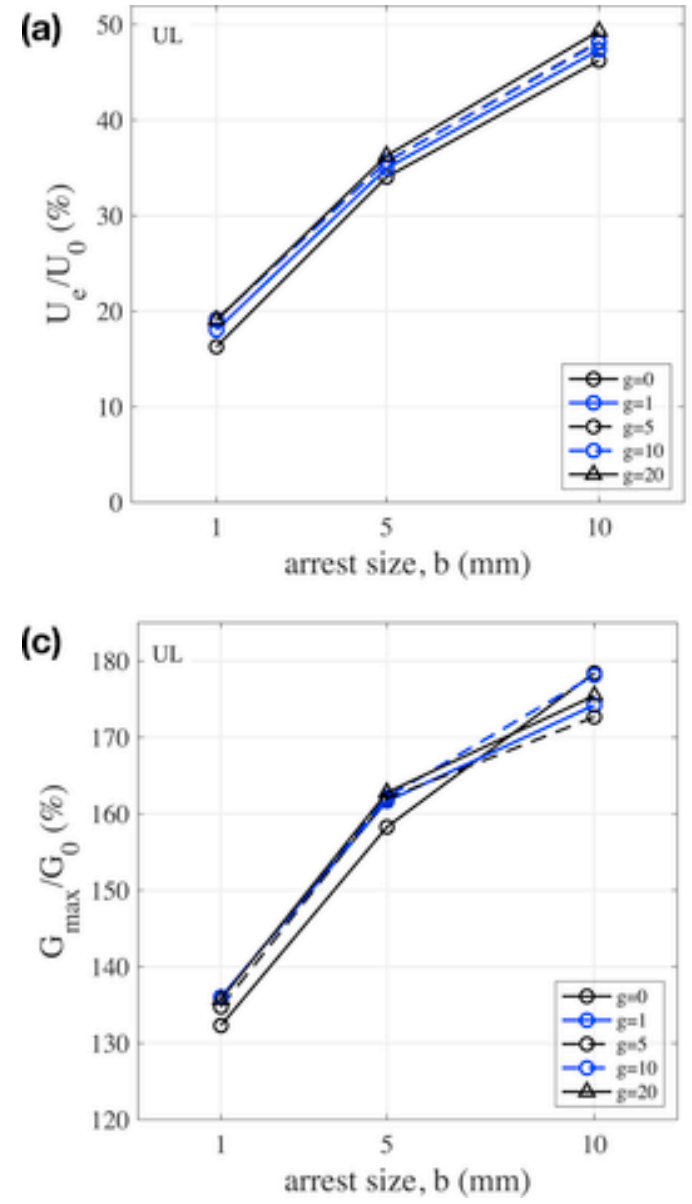

$U_{e}=U-U_{0}$. In the case of $U L$, the focus is only on the initiation and the detachment of the adhesive ligament from the arrest region. This choice of $U_{e}$ appropriately represents the initial enhancement of the crack-arrest feature. Since the ERR kept increase in the case of $U L$, the peak value $G_{\max }$ was obtained before the fully detachment of the adhesive ligament from the arrest region, and then it was normalized by the baseline toughness value $G_{0}$.

The effects of geometrical parameters of $U L$ and $B L$ when fracture toughness of the arrest region was fixed $\left(G_{a}=0.18 \mathrm{~kJ} / \mathrm{m}^{2}\right)$ are shown in Fig. 9. The results illustrated a same trend in the extra dissipated energy and the peak ERR. In the case of $U L$, since the ligament detached from the arrest region, it become clear that the anchor size $b$ played an important role, while the gap distance gave a secondary contribution. For example, the extra dissipated energy ratio $U_{e} / U_{0}$ increased from $19 \%$ to $48 \%$ and $G_{\max } / G_{0}$ from $133 \%$ to $180 \%$ as $b$ increased with $g=10 \mathrm{~mm}$ in $U L$, whereas they stayed constant as $g$ increased with $b=5 \mathrm{~mm}$, as shown in Fig. 9 (a). On the other hand, the anchor size was not significant but a proper gap distance was preferred in $B L$, because a short gap distance limited the ligament from detaching from the top CFRP/adhesive interface, which constrained the length of the adhesive ligament. In the case of $b=5 \mathrm{~mm}$ in $B L$, the extra dissipated energy ratio $U_{e} / U_{0}$ increased from $13 \%$ to $23 \%$, and then stayed constant around $23 \%$ when $g>1 \mathrm{~mm}$; while $G_{\max } / G_{0}$ improved from $127 \%$ to $140 \%$, and then stayed constant around $140 \%$ when $g>1 \mathrm{~mm}$, as shown in Fig. 9 (b). These observations are in accordance with the mechanisms discussed previously in Figs. 6 and 7. Interestingly, the first arrest region located at
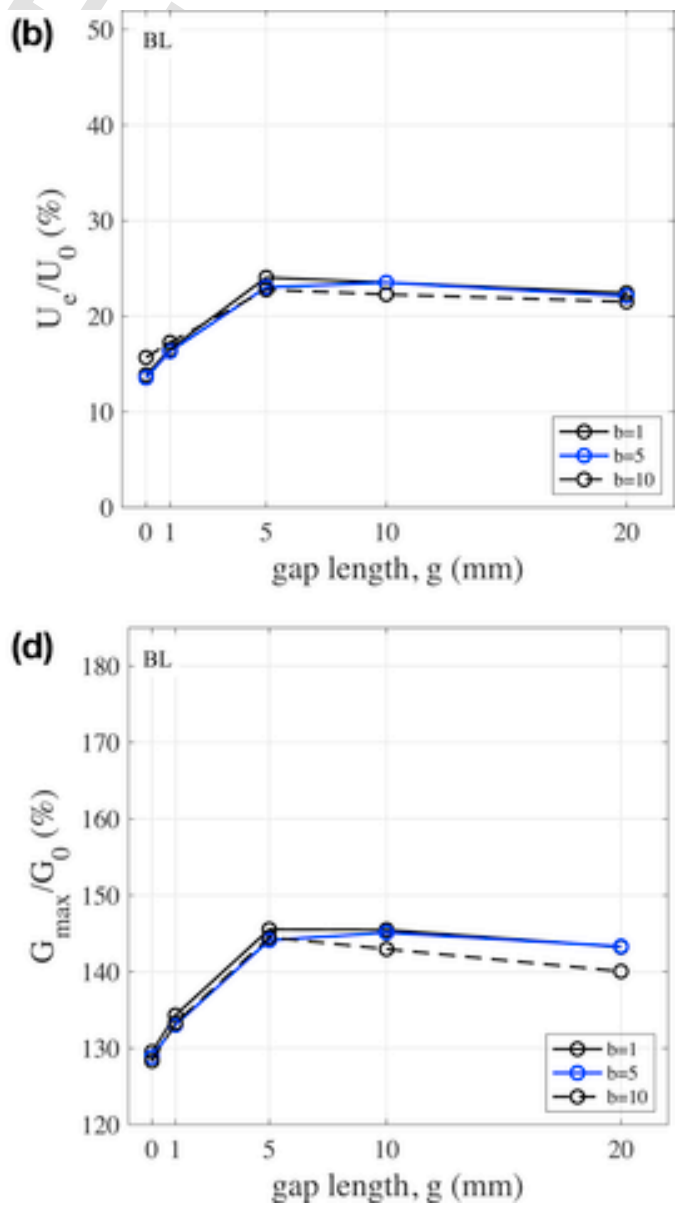

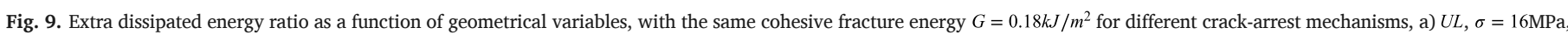

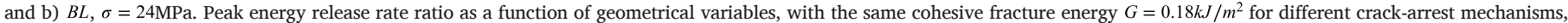
c) $U L, \sigma=16 \mathrm{MPa}$, and d) $B L, \sigma=24 \mathrm{MPa}$. 

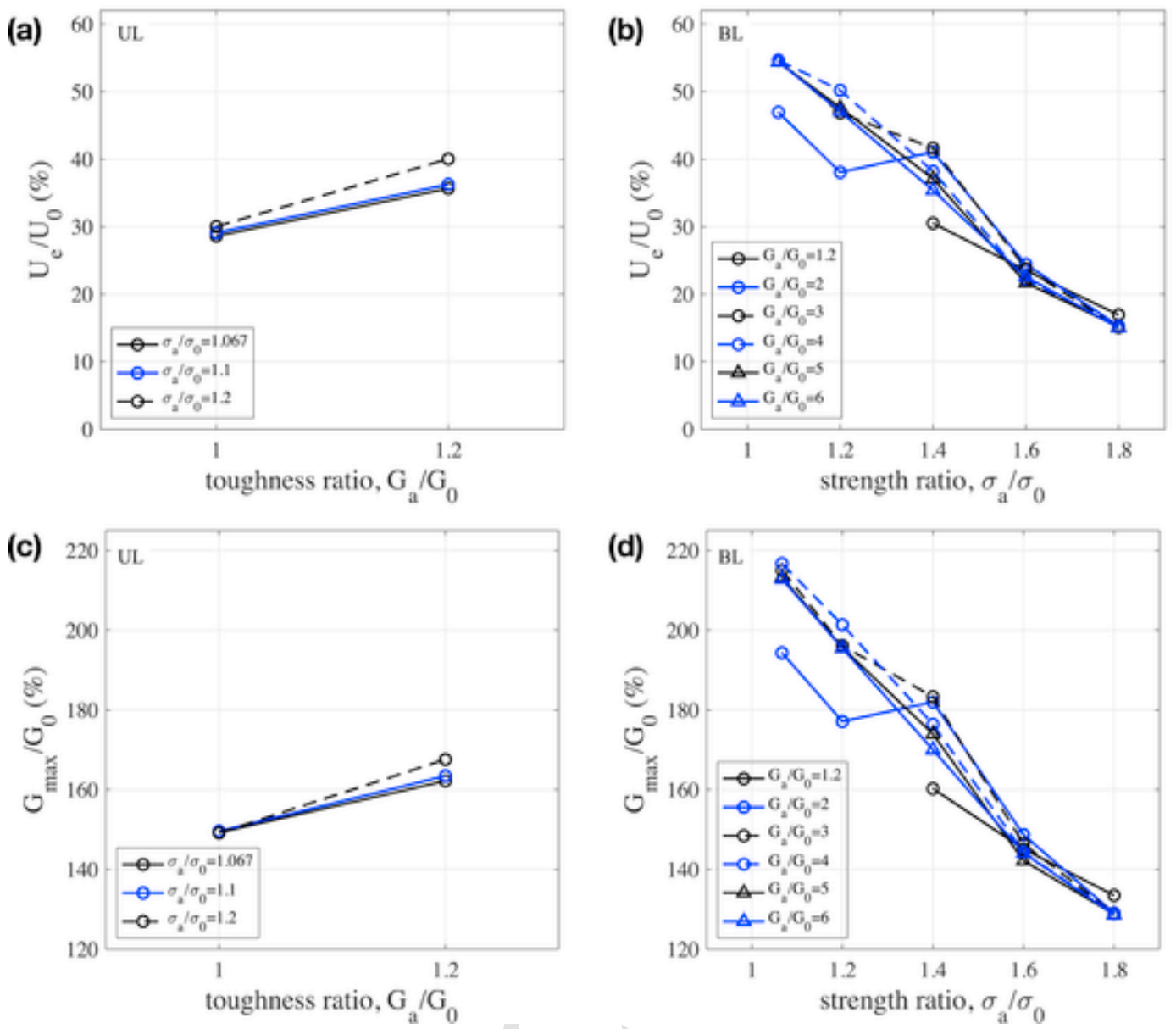

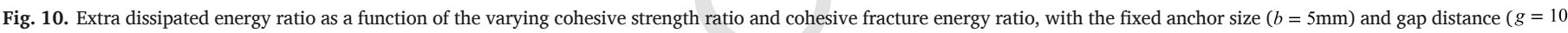

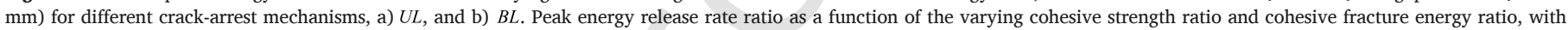
the fixed anchor size $(b=5 \mathrm{~mm})$ and gap distance $(g=10 \mathrm{~mm})$ for different crack-arrest mechanisms, c) $U L$, and d) $B L$.

the top CFRP/adhesive interface contributed very little. Only one arrest region at the bottom interface was enough to trigger the adhesive ligament either in $U L$ or $B L$, depending on its cohesive strength and cohesive fracture energy.

The variation of the extra dissipated energy and the peak ERR were also the same when changing the strength and toughness ratio between the arrest region and the baseline interface, while keeping the anchor size $(b=5 \mathrm{~mm})$ and the gap distance $(g=10 \mathrm{~mm})$ unchanged (Fig. 10). Due to the strict requirements of $U L\left(G_{a} / G_{0}<1.4\right)$, only six simulation data points can be obtained (Fig. 10 (a)). In this case, the ratio of fracture toughness was more critical because the detachment from the arrest region was the major part of the extra energy dissipation. For example, the ratio $U_{e} / U_{0}$ increased from $29 \%$ to $36 \%$ and $G_{\max } / G_{0}$ improved from $147 \%$ to $160 \%$, as $\sigma_{a} / \sigma_{0}=1.1$ in $U L$ (Fig. 10 (a)). On the other hand, the strength ratio $\sigma_{a} / \sigma_{0}$ was more significant in $B L$ because the ligament broke before detaching from the bottom interface where larger cohesive strength led to a stiffer arrest region. In the case of $G_{a} / G_{0}=4$ in $B L$, the extra dissipated energy ratio $U_{e} / U_{0}$ decreased from $55 \%$ to $15 \%$ and $G_{\text {max }} / G_{0}$ diminished from $220 \%$ to $127 \%$ (Fig. 10 (b)).

In summary, proposed patterning strategy was successful for introducing the crack-arrest feature by triggering the adhesive ligament, resulting in enhanced dissipated energy and applied load. However, the proposed patterning strategy in this study was simple; many other complex designs need to be investigated in the future. Moreover, the dissipated energy can be further enhanced by promot- ing the patterning strategy, with the aim to trigger multiple ligaments.

\section{Conclusions}

In this work, an adhesive ligament, acting as a crack-arrest feature, was simulated using the 2D finite element model by alternatively patterning the top and bottom interfaces of DCB specimens. A detailed parametric study was performed to understand the formation of the adhesive ligament and to investigate how the energy and ERR enhancement created by the crack arrest evolves by changing the anchor size, gap distance, cohesive strength, and cohesive fracture energy.

The 2D finite element model was validated and demonstrated identical responses compared to the conventional DCB simulation model. The proposed simulation model with double cohesive layers can be viewed as a more general method that provides further details on the failure of both interfaces and mechanical responses of the adhesive layer. The simulation results illustrate that the anchor size and gap distance had no effect on triggering the ligament and distinguishing between $U L$ and $B L$. The anchor size $b$ and fracture toughness ratio $\sigma_{a} / \sigma_{0}$ played a more critical role in enhancing the dissipated energy and ERR in $U L$, while gap distance $g$ and $G_{a} / G_{0}$ were more important in $B L$.

In summary, the proposed strategy was shown to be very promising as it may allow to increase the dissipated energy and hinder crack propagation. More interestingly, our results indicate that only one arrest interface with proper interfacial properties in the path of 
the crack surface would be enough to arrest the crack propagation and trigger the ligament. This would simplify positioning concerns between substrates. Of course, more advanced patterning strategies can be imagined using patterning of both substrates. This would become especially interesting to target multiple bridging ligaments, further increasing the dissipated energy and ERR of the joint. A more interesting idea is to modify the material properties of the adhesive layer to prevent its early-stage breakage. More plastic energy before failure, due to the stretching of the adhesive layer, can be dissipated, when a more ductile material was employed. In this way, the total energy dissipation can be largely improved (as large as $2 G_{0}$ ) and the safety of adhesively bonded CFRP joints can be ensured. Moreover, the effect of the loading condition is another interesting direction that deserves attention. As Daghia and Cluzel showed, different loading conditions led to different local failure mechanisms [40]. Thus, the proposed patterning strategy should be also investigated under the mode II loading condition.

\section{CRediT authorship contribution statement}

Ran Tao: Conceptualization, Methodology. Xiaole Li: Writing - original draft, Writing - review \& editing. Arief Yudhanto: Writing - original draft, Writing - review \& editing. Marco Alfano: Writing - original draft, Writing - review \& editing. Gilles Lubineau: Supervision, Conceptualization, Methodology.

\section{Declaration of competing interest}

The authors declare that they have no known competing financial interests or personal relationships that could have appeared to influence the work reported in this paper.

\section{Acknowledgement}

The research reported in this publication was supported by funding from King Abdullah University of Science and Technology (KAUST) Office of Sponsored Research (OSR) under award number OSR-2017-CRG6-3388.01.

\section{Appendix A. Supplementary data}

Supplementary data to this article can be found online at https://doi. org/10.1016/j.compscitech.2019.107964.

\section{Additional information - competing interests}

The authors declare no financial or non-financial competing interests.

\section{Data availability}

The datasets generated and/or analyzed during the current study are available from the corresponding author on reasonable request.

\section{References}

[1] T. Schmid Fuertes, T. Kruse, T. Körwien, M. Geistbeck, Bonding of CFRP primary aerospace structures, a discussion of the certification boundary conditions and related technology fields addressing the needs for development, Compos. Interfac. 22 (8) (2015) 795-808.

[2] P. Galvez, A. Quesada, M. Martinez, J. Abenojar, M. Boada, V. Diaz, Study of the behaviour of adhesive joints of steel with CFRP for its application in bus structures, Compos. B Eng. 129 (2017) 41-46.

[3] D. Markatos, K. Tserpes, E. Rau, S. Markus, B. Ehrhart, S. Pantelakis, The effects of manufacturing-induced and in-service related bonding quality reduction on the mode-I fracture toughness of composite bonded joints for aeronautical use, Compos. B Eng. 45 (1) (2013) 556-564.
[4] K. Katnam, L. Da Silva, T. Young, Bonded repair of composite aircraft structures: a review of scientific challenges and opportunities, Prog. Aerosp. Sci. 61 (2013) $26-42$.

[5] K. Tserpes, D. Markatos, K. Brune, M. Hoffmann, E. Rau, S. Pantelakis, A detailed experimental study of the effects of pre-bond contamination with a hydraulic fluid, thermal degradation, and poor curing on fracture toughness of composite-bonded joints, J. Adhes. Sci. Technol. 28 (18) (2014) 1865-1880.

[6] J. Korta, A. Mlyniec, T. Uhl, Experimental and numerical study on the effect of humidity-temperature cycling on structural multi-material adhesive joints, Compos. B Eng. 79 (2015) 621-630.

[7] A. Korayem, C. Li, Q. Zhang, X. Zhao, W. Duan, Effect of carbon nanotube modified epoxy adhesive on CFRP-to-steel interface, Compos. B Eng. 79 (2015) 95-104.

[8] R. Tao, M. Alfano, G. Lubineau, Laser-based surface patterning of composite plates for improved secondary adhesive bonding, Compos. Appl. Sci. Manuf. 109 (2018) 84-94.

[9] K. Tserpes, G. Peikert, I. Floros, Crack stopping in composite adhesively bonded joints through corrugation, Theor. Appl. Fract. Mech. 83 (2016) 152-157.

[10] P. Chang, A. Mouritz, B. Cox, Properties and failure mechanisms of pinned composite lap joints in monotonic and cyclic tension, Compos. Sci. Technol. 66 (13) (2006) 2163-2176.

[11] K.T. Tan, N. Watanabe, Y. Iwahori, Experimental investigation of bridging law for single stitch fibre using interlaminar tension test, Compos. Struct. 92 (6) (2010) 1399-1409.

[12] A. Yudhanto, G. Lubineau, I.A. Ventura, N. Watanabe, Y. Iwahori, H. Hoshi, Damage characteristics in $3 \mathrm{~d}$ stitched composites with various stitch parameters under in-plane tension, Compos. Appl. Sci. Manuf. 71 (2015) 17-31.

[13] S. Minakuchi, Fiber-reinforcement-based crack arrester for composite bonded joints, Proceedings of the 20th International Conference on Composite Materials, 2015

[14] T. Löbel, D. Holzhüter, M. Sinapius, C. Hühne, A hybrid bondline concept for bonded composite joints, Int. J. Adhesion Adhes. 68 (2016) 229-238.

[15] R. Tao, M. Alfano, G. Lubineau, In situ analysis of interfacial damage in adhesively bonded composite joints subjected to various surface pretreatments, Compos. Appl. Sci. Manuf. 116 (2019) 216-223.

[16] S. Heide-Jørgensen, S.T. de Freitas, M.K. Budzik, On the fracture behaviour of CFRP bonded joints under mode I loading: effect of supporting carrier and interface contamination, Compos. Sci. Technol. 160 (2018) 97-110.

[17] K. Maloney, N. Fleck, Toughening strategies in adhesive joints, Int. J. Solids Struct. 158 (2019) 66-75.

[18] L. Burns, A.P. Mouritz, D. Pook, S. Feih, Bio-inspired hierarchical design of composite t-joints with improved structural properties, Compos. B Eng. 69 (2015) 222-231.

[19] R. Akrami, S. Fotouhi, M. Fotouhi, M. Bodaghi, J. Clamp, A. Bolouri, High-performance bio-inspired composite t-joints, Compos. Sci. Technol. 184 (2019) 107840.

[20] E.P. Chan, A. Dongchan, A.J. Crosby, Adhesion of patterned reactive interfaces, J. Adhes. 83 (5) (2007) 473-489.

[21] M. Budzik, J. Jumel, K. Imielińska, M. Shanahan, Fracture in composite/aluminium joints of variable adhesive properties, J. Adhes. 85 (10) (2009) 736-754.

[22] S. Patinet, L. Alzate, E. Barthel, D. Dalmas, D. Vandembroucq, V. Lazarus, Finite size effects on crack front pinning at heterogeneous planar interfaces: experimental, finite elements and perturbation approaches, J. Mech. Phys. Solids 61 (2) (2012) 311-324.

[23] S. Xia, L. Ponson, G. Ravichandran, K. Bhattacharya, Adhesion of heterogeneous thin films II: adhesive heterogeneity, J. Mech. Phys. Solids 83 (2015) 88-103.

[24] C. Cuminatto, G. Parry, M. Braccini, A model for patterned interfaces debonding-application to adhesion tests, Int. J. Solids Struct. 75 (2015) 122-133.

[25] S. Heide-Jørgensen, M.K. Budzik, Effects of bondline discontinuity during growth of interface cracks including stability and kinetic considerations, J. Mech. Phys. Solids 117 (2018) 1-21.

[26] S.R. Ranade, Y. Guan, R.B. Moore, J.G. Dillard, R.C. Batra, D.A. Dillard, Characterizing fracture performance and the interaction of propagating cracks with locally weakened interfaces in adhesive joints, Int. J. Adhesion Adhes. 82 (2018) 196-205.

[27] R. Malkin, R.S. Trask, I.P. Bond, Control of unstable crack propagation through bio-inspired interface modification, Compos. Appl. Sci. Manuf. 46 (2013) $122-130$.

[28] Y. Xu, Y. Singh, C. Pan, G. Subbarayan, Adhesive toughness and instability in bonded heterogeneous films, Int. J. Solids Struct. 169 (2019) 41-54.

[29] P.W. Harper, S.R. Hallett, Cohesive zone length in numerical simulations of composite delamination, Eng. Fract. Mech. 75 (16) (2008) 4774-4792.

[30] E. Farmand-Ashtiani, J. Cugnoni, J. Botsis, Specimen thickness dependence of large scale fiber bridging in mode $\mathrm{i}$ interlaminar fracture of carbon epoxy composite, Int. J. Solids Struct. 55 (2015) 58-65.

[31] M. Alfano, G. Lubineau, G.H. Paulino, Global sensitivity analysis in the identification of cohesive models using full-field kinematic data, Int. J. Solids Struct. 55 (2015) 66-78

[32] R. Sills, M. Thouless, Cohesive-length scales for damage and toughening mechanisms, Int. J. Solids Struct. 55 (2015) 32-43.

[33] X. Wei, H. Wang, H.-S. Shen, A novel approach for the interfacial stress analysis of composite adhesively bonded joints, Compos. Sci. Technol. 184 (2019) 107830.

[34] L.P. Canal, M. Alfano, J. Botsis, A multi-scale based cohesive zone model for the analysis of thickness scaling effect in fiber bridging, Compos. Sci. Technol. 139 (2017) 90-98. 
[35] T. Suzuki, R. Matsuzaki, A. Todoroki, Y. Mizutani, Crack growth analysis of a composite/adhesive interface toughened by in-mold surface preparation, Int. J. Adhesion Adhes. 42 (2013) 36-43.

[36] P. Liu, Z. Hu, S. Wang, W. Liu, Finite element analysis of the void growth and interface failure of ductile adhesive joints, J. Fail. Anal. Prev. 18 (2) (2018) 291-303.

[37] S. Jain, K.M. Liechti, R.T. Bonnecaze, Cohesive zone models to understand the interface mechanics of thin film transfer printing, J. Appl. Phys. 125 (7) (2019) 075301.

[38] A.Z. Haider, X.L. Zhao, R. Al-Mihaidi, Mechanical behaviour of normal modulus carbon fibre reinforced polymer (CFRP) and epoxy under impact tensile loads, Procedia Eng. 10 (2011) 2453-2458.
[39] Standard Test Method for Mode I Interlaminar Fracture Toughness of Unidirec tional Fiber-Reinforced Polymer Matrix Composites, ASTM Internat, 2014.

[40] F. Daghia, C. Cluzel, The climbing drum peel test: an alternative to the double cantilever beam for the determination of fracture toughness of monolithic laminates, Compos. Appl. Sci. Manuf. 78 (2015) 70-83.

[41] J. Williams, End corrections for orthotropic dcb specimens, Compos. Sci. Technol. 35 (4) (1989) 367-376. 